\title{
Learning for Competence through Distance Education
}

\author{
Natalya Yelashkina, ${ }^{1, *}$, and Oksana Kaverina ${ }^{2}$ \\ ${ }^{1}$ Irkutsk State National Research Technical University, Irkutsk, Russia \\ ${ }^{2}$ Irkutsk State University, Institute of Filology, Foreign Languages and Mediacommunications, Irkutsk, Russia
}

\begin{abstract}
The article focuses on the content components necessary for the formation of a special learning competence for distance-learning students. The authors propose their own interpretation of the constituents of the learning competence with due regard for the specificity of studying foreign languages by means of telecommunication technologies. Such components are to be considered in the context of the learnercentered paradigm. The content of the technology created includes knowledge of the essence and specificity of learning activities in foreign language acquisition. The article demonstrates a big variety of terms in the methodical literature. The authors describe learning skills and say that they are such ways of learning activities that do not directly constitute a mechanism for the formation of a communication competence, but rationalize this process. The article helps to understand that studying foreign languages with the help of telecommunications presents a number of difficulties and it is proved that learning competence remove the difficulties of distance learning. The article gives the important methodological provisions defining a role and the place of learning competence of a context of distance language learning.
\end{abstract}

It has become a commonplace to see any complex didactic phenomenon in terms of its components knowledge, skills and experience based on creative activity as well as the emotional and volitional attitude. In accordance with the modern learner-centered paradigm in the system of education, these components are aimed at forming the experience of the individual, created through the acquisition of social experience of humankind, enshrined in material and spiritual culture [1].

As applied to the competence approach in teaching foreign-language communication, the acquisition of such experience has priority importance in this regard. It means a shift from knowledge-based methods [2] focusing on knowledge as the center of the methodological universe and overshadow the person himself. Today, profound personal substructures of students must be changed: it is necessary to form their readiness and ability to socially demanded activities. In other words, the content components of modern education are based entirely on the category of "personal experience" [3].

This experience comprises the following areas: a) personal experience, differentiated according to the theory-practice principle (knowledge and abilities); b) personal experience, differentiated on the basis of creativity in educational activity (reproductive and consistently increasing levels of creative activity) [1].

Thus, the content component of the technology for the formation of students' competence should include:

\footnotetext{
* Corresponding author: Nat.Yelashkina@gmail.com
}

- knowledge of the nature and peculiarities of learning activities in foreign language acquisition, its focus on improving the learning process of foreignlanguage communication, the importance of its optimization;

- learning skills special for the subject "Foreign Language" and for distance learning students-future linguists which break up into a) the skills of reproductive learning activities and b) the skills of creative learning activities.

In all learning theories, the task of students is to acquire knowledge, skills and attitudes that are needed or desired, so the explication of any competence through the concepts listed above is quite traditional in linguodidactics. We define knowledge as the theoretical or practical understanding of subject. Skills are the proficiencies developed through training or experience, which are usually something that has been learned.

We follow this tradition, detailing the given conceptual interpretation of learning competence and its components. It is these components that make up its internal operational content. In the context of the technology of learning that we create, they will be considered as components of the content of teaching students effective learning activities to learn foreign language communication. Let us consider these components in detail.

Knowledge, as a content component, is called to form an indicative basis of any activity, to create some information background (similar to "background 
knowledge"), favorably facilitating to assimilate this activity by students. In the context of the formation of learning competence, judging by its special features, a peculiar set of knowledge is needed.

When revealing the knowledge component of the content, we refer to the research study of E.G. Tareva as a methodological framework aimed at improvement, efficiency enhancement (rationalization) of students' learning activity [4]. The author provides a thorough analysis of the set of knowledge, which is necessary to build a rational learning style. On carrying out the specification relevant for our work, we have established the knowledge necessary for the formation of the students' learning competence in the distance learning mode, which is as follows:

- the knowledge of the peculiarities of foreignlanguage acquisition in conditions of territorial remoteness of students from teachers;

- the knowledge of the peculiarities of technologymediated language learning;

- the knowledge of the importance of rational (economical, effective, emotionally positive) implementation of learning activities to achieve the effectiveness of distance learning (DL);

- the knowledge of the importance of interactivity in DL;

- the knowledge about the difficulties of DL in foreign language acquisition and the ways to overcome them;

- the knowledge about the functions of a distancelearning teacher;

- the knowledge of the specifics of distance-learning activities;

- the knowledge of the need to actualize students' learning experience in technology-mediated language learning of foreign-language communication;

- the knowledge of the importance of using previously accumulated experience of foreign language learning;

- the knowledge of a creative approach to the formation of students' methods of learning activity;

- the knowledge of the need to the develop students' system of priority training activities when learning a foreign language in conditions of DL.

This set of knowledge should serve as the basis for the formation of the students' positive emotionalvolitional attitude to learning activity, awareness of its importance, creating an internal motivation for the implementation of learning. This motivation, as we know, directly correlates with a specifically cognitive, intellectual need, characterized by a positive emotional background (L.I. Bozhovich, I.A. Zimnyaya, YU.M. Orlov, P.M. Yakobson, etc.).

The next component of the technology content of the formation of students' learning competence is skills. We think it necessary to specify the components of the skills relevant to acquiring the learning competence that function as its operational components to be learnt by students through distance education. For the purposes of this paper, we will use the term learning skills though they are also often called study skills or key skills. These skills, which are needed for study and can be gained as a result of study, are preferably to be included in the learning outcomes for any distance-learning course.

Thus, our task is to establish a list of learning skills that make up the learning competence, the peculiarities of which are determined by distance learning. We will hereafter systemize and classify these phenomena, determining the connections and dependencies that we will establish according to the grounds identified. The complexity of this research lies in the fact that nowadays there are many approaches to the identification of learning skills, so consequently no unified opinion can be stated. We will try to justify our judgment on this issue.

To establish a list of learning skills, the content of which is determined by the peculiarities of distance education, it is first of all necessary to analyze the views on the specific features and range of learning skills existing in methodological science.

The literature review shows that learning skills have been under intensive study (E.V. Apanovich, 2003; A.L. Berdichevskiy, 1989; I.L. Bim, 2001; V.P. Bojko, 1993; M.M. Gohlerner, 1979; V.M. Gurlenov, 1992; G.V. Ejger, 1991; A.V. Konysheva, 2005; N.F. Koryakovceva, 2002; B.A. Lapidus, 1970, 1982; G.N. Nefedova, 1995; F.M. Rabinovich, 1988; P.A. Razinov, 1989; G.V. Rogova, 1984, 2000; V.V. Safonova, 1996; E.G. Skosorenko, 1993; M.N. Sosyak, 1989; E.G. Tareva, 2002; C. Faerch \& G. Sasper, 1980; H. Holec, 1997; R. Oxford, 1989, 1990 etc.).

Practically all research papers unequivocally state the importance of learning skills to improve, optimize, and rationalize the educational and cognitive processes of foreign language acquisition. According to B.A. Lapidus, it is the development of the learning skills in the initial stage of education that can ensure the greatest efficiency of learning in future and might be a crucial learning strategy [5]. P.A. Razinov claims that the lack of general and special learning skills leads to a decrease in cognitive motivation, contribute to the fact that students lag behind in their studies. Besides, the lack of general and special learning skills is one of the main reasons for the improper use by students of a foreign language as a means to "go into the world culture and scientific and technological progress" [6].

G.V. Rogova and I.N. Vereshagina are more categorical in their statements. They consider that if students are not provided with rational learning techniques they will fail to learn a foreign language even at the level of the requirements formulated by the curriculum [7]. Holec reasonably believes that "without the formed complex of learning skills in mastering a foreign language, it is extremely difficult to predict success" [8].

Despite the general opinion of scientists about the significance of learning skills in the process of language learning, the analysis of the above-listed works proves that there is a clear ambiguity in judgments about the essence of learning skills and their nomenclature in the methodical literature. This ambiguity, first of all, manifests itself in the terminology used by scientists. 
One of the central problems is that there are a number of terms used in the methodical and linguodidactic literature to describe the term "learning skills" [4]. These terms include "learning methods" [5], "learning labour" [9], "language learning strategy" [10] and others.

Since a variety of terms are used to describe the concept of learning skills in the methodical literature it entails the confusion and ambiguity over its meaning and essential characteristics as well as it leads to diffuseness of the term itself. This is described in detail in the works of modern scientists. Briefly, the essence of their arguments is reduced by the fact that the learning skills special for the subject "Foreign Language" are either replaced by intellectual skills (in the terminology of I.Ya. Lerner) [11], or they are identified with verbal and communication skills, or general educational skills.

We completely agree with the critical view on this issue. The critical analysis as we see it helps to understand the reasons of such terminological confusion, which can lead away from a true and productive solution to the problem of the formation of learning competence.

The above-mentioned trends are confirmed by the research paper of A.V. Konysheva (2015). The author outlines the following components of learning labour, which are necessary to organize self-directed learning of a foreign language:

- the skills to set goals;

- the skills to plan and assess one's learning activity;

- the skills to concentrate psychic and physical forces to achieve the goal;

- the skills to be responsible for the right option of the task;

- the skills to time and measure the studied material [9].

As you can see, these skills directly correlate with the characteristics of the previously considered students' individualization and autonomy in language learning. Indeed, the characteristic feature of autonomy in the representation of the listed authors is that students are to take responsibility for all the stages of educational and cognitive activities: from choosing the goal to determine the content and sequence of methods and techniques used to evaluating the result. With regard to the characterization of autonomy as such, this list of skills could seem quite appropriate to the purpose of this research paper. However, the content of the learning skills given above concentrates on language learning and teaching in very general terms, without taking account of the specific features of the subject "Foreign Language" [12].

Accordingly, such components, which can determine the peculiarities of learning competence in general, are not enough for the formation of the special learning competence for distance education. They are only indirectly able to influence the choice of certain learning skills required in terms of implementation and rationalization of language learning which is under consideration (distance education).

In the research paper of N.D. Galskova, N.I. Gez (2004) the authors don't aim to distinguish either general learning skills or the special learning skills for language learning. Nevertheless, they emphasize the cognitive aspect in the process of language learning. The researchers, in particular, claim that "the effectiveness of language and culture acquisition is determined by the degree of students' development of their learning skills. The students should be able to:

1) organize their learning activity (for example, to work individually, in pairs or groups, or as a whole class; to check, to evaluate and correct their work or the work of a fellow student, etc.);

2) activate intellectual processes (for example, to recognize a particular language phenomenon, to compare this phenomenon with the analogous one in one's native language, etc.);

3) prepare for the learning process and actively participate in it (for example, to make notes, to make a plan, to use a dictionary, etc.);

4) organize communication activities (for example, to plan utterances ahead, to provide time for students to formulate their thoughts into explicit language with a limited set of language tools, to use gestures and facial expressions in oral communication, etc.) [13].

The analysis of the skills suggested shows that only one of the groups of these skills has a truly educational meaning caused by the specificity of the subject "Foreign Language", namely, the second one. Groups of skills 1 and 3 have exclusively general educational purposes, whereas group 4 has a purely communication meaning and therefore, should be related to the communication skills.

Having designated our attitude to the proposed list of skills, we consider it necessary to justify our position that these skills, according to the authors, belong to the group of cognitive ones. We lean towards the idea that it is not entirely true and only partly makes sense because any human activity is necessarily accompanied by cognitive processes. Therefore, any skill that is actualized by a person can be characterized as cognitive. At the same time, the educational character of the learning skills under discussion becomes fuzzy. Thus, we cannot agree to call the methods of educational activity "cognitive" or "metacognitive" because of the ambiguity of the content that is put into these concepts.

The same trend can be observed in the research paper of I.L. Bim (2001), where the general educational skills (to work with a textbook / workbook, mastering the technology of performing the most common types of exercises, tests; to independently search for and select additional materials on the topic) adjoin the proper communication skill (to use the methods of active speech interaction), as well as with the special educational skills (to develop one's own strategy to learn vocabulary - to make up and keep a vocabulary notebook, make up a glossary of German-English equivalents, keep a word dairy, build word families, etc., in order to better acquire new vocabulary, to be able to apply grammar rules to building new grammar patterns, to make generalizations based on some observation, analysis of textbooks as well as the teacher's speech) [14].

The similar tendency can be seen in the textbook of A.N. Shchukin, in which, among the tasks that are 
intended to be solved in the course of teaching a foreign language, he calls general-purpose tasks such as to develop the skills: to use a textbook / dictionary and technical teaching aids, to take notes and make up logical statements, to use translation [15]. It is not difficult to conclude that this set of skills does not have a specific educational purpose.

I.D. Trofimova, solving the problem of the formation of autonomous reading strategies states that students' skills to build their own variants of behavior in reading directly depends on their acquisition of not only a complex of educational and communication skills, but also reflection [16]. The author offers a set of communication, strategic (compensatory), general educational skills, relevant for the activity of autonomous reading. In her paper the researcher highlights the need for students to have the skills of a broad educational and narrow special character. However, with the specification of this list, only the general educational skills are given, whereas special educational skills are not adequately reflected in the work of the scientist.

Thus, the methodological studies of recent years provide a far from perfect list of learning skills. Its incompleteness, as we can see, lies in the fact that the educational essence of these concepts is not fully realized by researchers. Consequently, the list of learning skills directly depends on their contents, and the imperfection of the second automatically leads to the drawbacks of the first.

Following the conclusion above, we think it relevant to formulate the special learning skills necessary for language acquisition in the form of distance education which we will use below. We take into consideration:

- the methodological aspect of learning skills interpretation;

- the research works of (E.G. Tareva), who studied the specifics of educational skills, special for the subject "Foreign Language";

- the concept of "learning competence", the constituents of which the learning skills are;

- the specificity of distance education for students learning languages.

The learning skills which are necessary for distancelearning students are the methods of learning activities that do not directly constitute a mechanism for the formation of a foreign communication competence, but rationalize this process, eliminating the essential difficulties of distance education. This is a didactic form of concretizing the learning competence of distancelearning students, the latter, due to its specificity and purpose, increases the effectiveness of the process of forming a secondary linguistic personality - an integrated personal trait consisting of a set of special knowledge and skills allowing students to carry out linguistic and intercultural communication through distance education.

In order to proceed to the list of special learning skills necessary in the conditions of the distance education, we find it necessary to rely on the nomenclature of these skills in literature. This nomenclature closely corresponds to the definition of learning skills formulated by us. In other words, this is a list, which includes skills corresponding to the following parameters:

a) they do not constitute the process of the formation of communicative competence (they do not directly relate to the content), but optimally organize this process;

b) they correspond to the specificity of the subject "Foreign Language" and, as a consequence, do not work for the organization of learning activity of other subjects, and therefore, they are not general learning skill;

c) they are focused on the rationalization of all aspects of the learning process for students to be able to communicate in a foreign language: the procedures of acquisition of linguistic knowledge, the formation and development of various types of speech activity, the acquisition of socio-cultural knowledge as linguistic knowledge is embedded in and constitutes socio-cultural knowledge through the use of language in authentic activity which mediates between linguistic and sociocultural knowledge, etc.;

d) they meet the requirements of distance education;

e) they are notably different from the similar system, formed (in many ways spontaneously) in a foreign language classroom of a secondary school.

The analysis above enables us to formulate new insights into the learning skills to be developed through distance education. To the list above there should be added special learning skills which can provide elearning success.

If communication is understood as an abundant exchange of information in a foreign language, then the most appropriate form of transmission of this information in remote communication via Internet is the perception / transmission of text (hypertext) messages. In other words, we deal with written communication as the ability to convey information from one person to another in written form. Let us consider in more detail its specific features which are bilateral in nature.

One side marks the process of perception and processing of information. This process is carried out through the use of reading skills, because most of the information received by the brain in the use of telecommunications as a means of learning comes through visual channels (eyes). Thus, reading can be as a speech (communicative) skill (as an end in itself), and as a means of learning (not as an end in itself). In the second case, it becomes a means of "serving" the process of communication in another language, the speed of its flow directly depends on the effectiveness of the formation / development of other speech skills, the formation of authentic automatisms of operating with linguistic, linguistic-cultural and culturally significant material. For example, when performing oral communication the communicant can quickly learn additional, explanatory, reference, sociolinguistic, sociocultural information intermittently switching to reading reference books, dictionaries, hypertexts, etc.). In addition, any student's learning activity will be effective if the student at a fast reading speed understands the essence of the tasks and instructions necessary for their performance. As we can conclude, in this perspective, fast reading becomes more educational in nature. 
To "strengthen" its educational effect, some additional parameters should be set for this skill. Once again, we emphasize that the effectiveness of the student's foreign-language activity will be largely determined by the speed characteristics of reading as a learning skill. The speed-reading skills, in our opinion, roughly correspond to the speed of familiarizing reading. The parameter "speed" is one of the most important for the operatively flowing mode of telecommunication interaction between communicants. The lack of properly developed speed-reading skills (in other words, delay in time due to slow reading) can lead to comprehension failure, negative / undesirable reaction of the partner in communication and even to the violation of the communicative act.

It should be noted that the means of computer telecommunications have the potential to achieve speed reading. The speed of reading should be accompanied by another parameter - "comprehension", which characterizes the content side of the reading process and indicates reaching a certain level of comprehension necessary for specific conditions of any learning activity. Speed in reading and comprehension work together. All speed reading techniques have one thing in common: a student avoids pronouncing and "hearing" each word in his head as he reads it, a process known as "subvocalization." Instead, students should "skim" lines or groups of words, as they can understand words more quickly than they can say them. Then students should focus on blocks of words rather than on individual ones.

Thus, the ability to read quickly should be included in the list of learning skills required for a student who is willing to be able to speak a target language in conditions of distance education.

The second side of written communication on the Internet concerns the process of information transfer, and, as it can be concluded, it is related to foreign writing activity, namely, the technical side of writing. By analogy with the previous arguments, we can draw a conclusion about the educational importance of the ability to write quickly when communicating via the Internet. For example, with the ability to write quickly, the communicant can quickly remove the lack of comprehension because he has the ability to send a request for a particular purpose can mark the most relevant information, take notes, etc.

Writing in such an educational context is very specific in view of the lack of the need for graphic and calligraphic representations of utterances. Another specific feature of writing is its speed. Writing should be as fast as reading. On the other hand, it is to be understandable. Thus, computer-mediated language learning requires the students to develop speed-writing skills.

Both speed reading skills and speed writing skills ensure the effectiveness of immersing students in a target language environment and, most importantly, through engaging them in real-world tasks (in the form of electronic dictionaries, reference books, encyclopedias, samples of musical, theatrical culture, virtual museums, virtual sightseeing tours, etc.). And on this basis, these learning skills make students be induced to speak the target language in everyday life. This possibility is completely based on the hypertext principle of information access. Thus, the efficiency of the process of on-screen perception and conceptualization of information in a foreign language, which is presented mainly in written form, is provided if a student has speed reading skills. In its turn, participation in telecommunication projects requires the communicant to have proper speed writing skills.

In a nutshell, speed reading and speed writing skills are central components of the system of special learning skills which constitute learning competence through distance education. It is assumed that, once the students have acquired these learning skills, they will be able to build real telecommunication via Internet. When learning takes place in an online environment, there is a set of learning skills required and expected, which enable students to best achieve personal and curricular objectives while studying in an online setting. The interpretation of the learning skills suggested follows modern trends and scientific priorities in linguodidactics.

\section{References}

1. V.S. Lednyov, Scientific education: development of abilities for scientific creativity 2, (MSAU, Moscow, 2002)

2. V.A. Slastyonin, I.F. Isaev, Y.N. Shiyanov, Pedagogics (Publishing centre "Academia", Moscow, 2002)

3. H. Wang, Interactivity in E-Learning (IGI Global, Dakota State University, USA, 2011)

4. E.G. Tareva, Innovation of linguodidactic technologies: super omnia veritas, Humanitarian educational environment of a technical university, 309-311 (2016)

5. B.A. Lapidus, Foreign languages at school 17, 518 (2010)

6. P.A. Razinov, Foreign languages at school 4, 8-15 (1989)

7. G.V. Rogova, I.N. Vereshchagina, Methods of teaching English at the initial stage in general education institutions (Prosvescheniie, Moscow, 1988)

8. H. Holec, I. Huttunen, Learner autonomy in modern languages Research and development (Council for Cultural Co-operation of the Council of Europe, Strasbourg, 1997)

9. A.V. Konysheva, Higher Education in Russia 11, 18-25, (2009)

10. R. Oxford, Language Learning Strategies. What Every Teacher should Know (Newbury House Publishers, New York, 1990)

11. I.Ya. Lerner, Reader 1, 357-365 (2012)

12. N.I. Almazova, V.Ya. Kikot, St. Petersburg State Polytechnical University Journal. Humanities and Social Sciences 3(179), 9-16 (2013) 
13. N.D. Galskova, N.I. Gez, Theory of Foreign Language Teaching: Linguodidactics and Methodics (Academia, Moscow, 2006)

14. I.L. Bim, The concept of teaching a second foreign language (German based on English) (Title, Obninsk, 2001)

15. A.N. Schukin, Teaching Foreign Languages: Theory and Practice (Filomatis, Moscow, 2006)

16. L.V. Trofimova, Bulletin of Tambov State University, Humanities 2(26), 124-128 (2002) 